\title{
Enrichment of Mutant KRAS Alleles in Pancreatic Juice by Subtractive Iterative Polymerase Chain Reaction
}

\author{
Carsten Fischer, Julia Büthe, Peter Nollau, Stephan Hollerbach, \\ Karsten Schulmann, Wolff Schmiegel, Christoph Wagener, and Peter Tschentscher \\ Department of Clinical Chemistry (CF, JB, PN, CW, PT), Clinic for Internal Medicine, University Hospital Eppendorf, \\ Hamburg; and Medical Clinic (SH, KS, WS), University Hospital Bochum, Bochum, Germany
}

SUMMARY: The detection of mutant tumor genes holds great promise for an early diagnosis of primary tumors and residual malignant disease. When few tumor cells are present with an excess of nonmalignant cells of the same lineage, the excess of wild-type alleles over mutant tumor alleles presents an analytical problem. The subtractive iterative PCR (siPCR) assay presents a new approach to solving this problem. To achieve an enrichment of mutant alleles, wild-type alleles are removed by differential hybridization to complementary oligonucleotides spanning the region of the gene in which point mutations are expected. The nonbound fraction is reamplified by PCR. By iterating this process, mutant alleles can be detected in the presence of an excess of wild-type alleles with high sensitivity. To prove the feasibility of siPCR, pancreatic juice samples were analyzed for KRAS mutations. Pancreatic juice obtained from patients with pancreatic carcinoma or chronic pancreatitis during endoscopic retrograde cholangiopancreatography was analyzed for point mutations in codons 12 and 13 of the KRAS gene. In each of six samples from tumor patients, mutations in codon 12 were detected. One of nine samples from patients with chronic pancreatitis scored positive. (Lab Invest 2001, 81:827-831).

$T$ he detection of mutant alleles of tumor genes in specimens such as urine, pancreatic juice, sputum, or stool holds great promise for an early diagnosis of cancer (Sidransky, 1997). In addition, the detection of mutant tumor genes in tissue samples such as lymph nodes or resection margins may allow a sensitive diagnosis of residual malignant disease. When few tumor cells are present with an excess of nonmalignant cells, mutant tumor alleles constitute a minor fraction of the total compared with wild-type alleles, so that a detection of point-mutated alleles presents a major analytical problem. So far, this problem has been solved only when the point mutations in the respective tumor genes are known a priori. Because KRAS is one of the tumor genes most frequently mutated in human tumors, most experience in the aforementioned diagnostic applications has been obtained using this oncogene as a target. Various methods have been described for detecting mutant KRAS alleles in the presence of an excess of wild-type alleles. These include detection of cloned PCR products by allele-specific oligonucleotide hybridization (Sidransky et al, 1992), digital PCR (Vogelstein and Kinzler, 1999), allele-specific PCR (Smith-Ravin et al, 1995), a modification of the oligonucleotide ligation assay, termed Point-EXACCT (Somers et al, 1994,

Received January 29, 2001.

Address reprint requests to: Dr. Christoph Wagener, Abteilung fir Klinische Chemie, Klinik fuir Innere Medizin, Universitätsklinikum Hamburg-Eppendorf, D-20251 Hamburg, Germany.E-mail:wagener@uke.uni-hamburg.de
1998), mutant-enriched PCR (Chen and Viola, 1991; Kahn et al, 1991), and a modification of the latter making use of a thermostable restriction enzyme that is included in the PCR reaction and cuts any amplification products derived from the wild-type sequence (Fuery et al, 2000). We conceived a method based on the subtraction of wild-type alleles by hybridization to complementary oligonucleotides. The nonbound fraction is reamplified and submitted to a second round of absorption. The technique, termed subtractive iterative PCR (siPCR), allows the detection of KRAS mutations in pancreatic juice with high sensitivity and reliability.

\section{Results}

The principle of the siPCR technique applied for the detection of mutant KRAS alleles in pancreatic fluid is shown in Figure 1A. The target sequence is amplified by PCR. Because one of the primers is biotinylated, single-stranded DNA (ssDNA) can be generated by binding the PCR product to streptavidin-coated magnetic particles followed by alkali denaturation. The ssDNA is loaded to a chromatographic device containing oligonucleotides that are complementary to the wild-type sequence. Wild-type DNA is retained on the column because it hybridizes to the immobilized oligonucleotides under stringent conditions. Mutated alleles are collected in the flow-through, reamplified by PCR for another column run, or analyzed by DNA sequencing. 
A

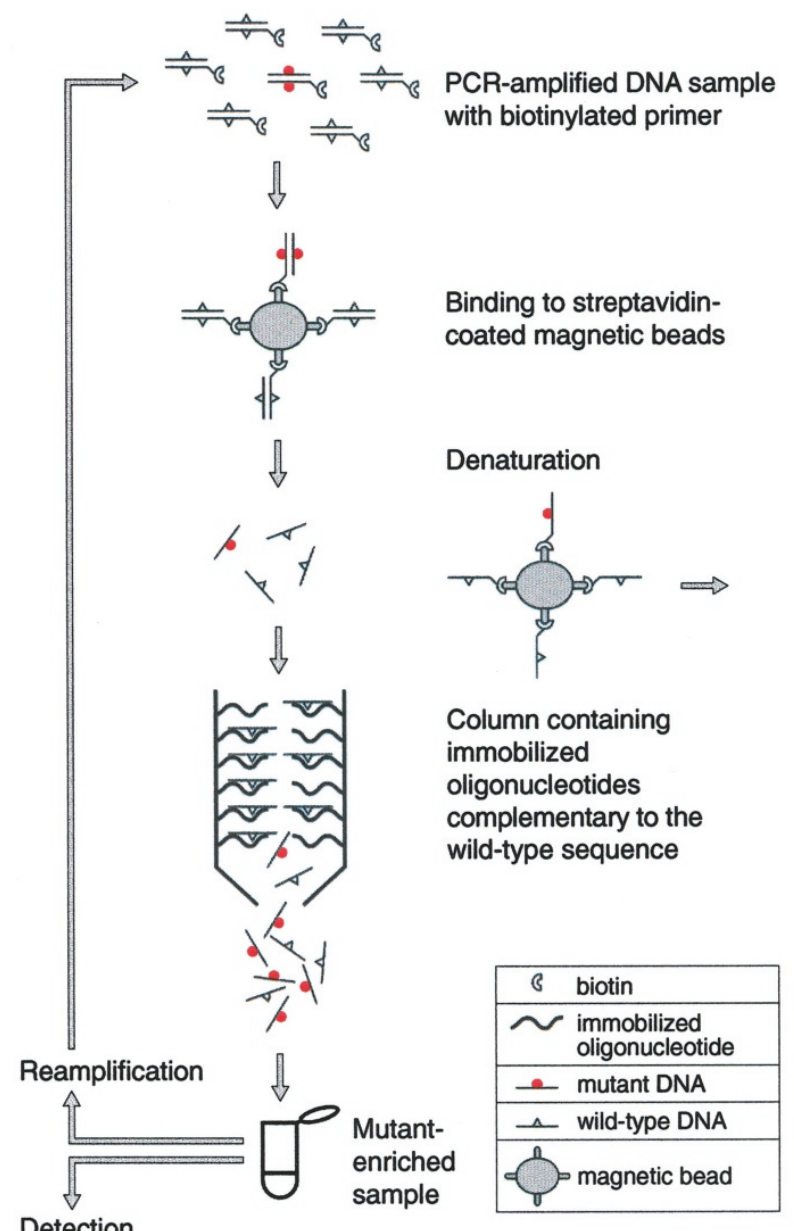

B

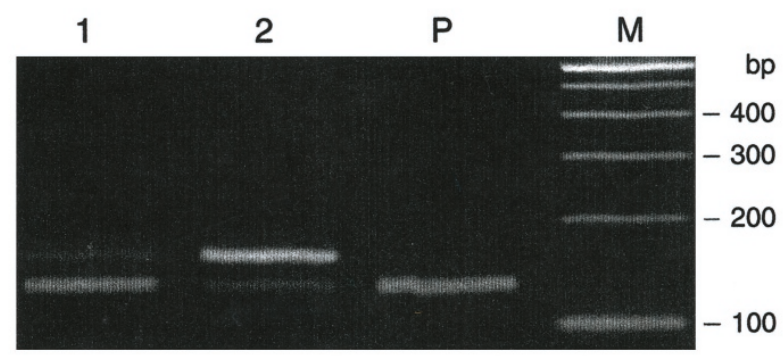

C

$13 \quad 12$

CTACG C CAC CAGG

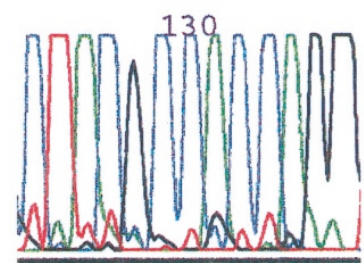

chronic pancreatitis bp

400

200

100

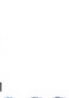

CTACGC CAC CAGG

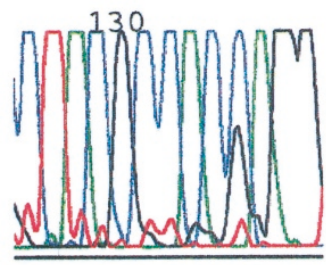

pancreatic carcinoma

Figure 1.

A, Schematic presentation of the subtractive iterative polymerase chain reaction (SiPCR) for the detection of mutant DNA in the presence of an excess of wild-type alleles. B, siPCR amplification and removal of wild-type alleles to enrich a mutant KRAS allele. Restriction fragment length polymorphism (RFLP) analysis of a 1:1000 mixture of mutant and wild-type alleles. 1, Eluted DNA after the first separation; 2, eluted DNA after the second separation; P, mixture before separation; M, size marker. C, Results of DNA sequencing (antisense) of the KRAS gene codons 12 and 13 in pancreatic juice after a two-step siPCR. 
For an evaluation of the siPCR assay, mutant DNA harboring a point mutation in codon 12 of the KRAS gene was used as a model system. A 1:1000 mixture of single-stranded mutant to wild-type KRAS alleles was loaded on a chromatographic device. A second PCR was performed on the eluted DNA; ssDNA was prepared and passed over an identical column. As shown in Figure 1B, only a faint band of the mutant allele is visible in the restriction fragment-length polymorphism (RFLP) gel after the first separation step (Lane 1). In contrast, the mutant allele exhibits a stronger signal than does the wild-type allele in the flow-through collected after the second chromatographic run (Lane 2). The result was confirmed by DNA sequencing.

To prove that a diagnostic siPCR using biological samples is feasible, we collected pancreatic juice during routinely performed endoscopic retrograde cholangiopancreatography from patients who were clinically suspected of a pancreatic disease. The samples were analyzed by applying the two-step siPCR protocol described above. Figure $1 \mathrm{C}$ shows typical examples of the outcome of a carcinoma sample and a chronic pancreatitis sample. An additional peak in the sequence analysis of the processed DNA indicates the presence of mutated KRAS alleles in samples obtained from carcinoma patients. The sample shown in the figure was analyzed in triplicate with each of the test results confirming the same specific base substitution. In contrast, no peak corresponding to a base substitution can be identified between the wild-type sequence and the background signals of the sequence reaction in the case of a chronic pancreatitis sample. No mutations in codon 12 or 13 were present in eight of nine samples from chronic pancreatitis patients (Table 1). One sample contained a mutation at the first position of codon 12. All six samples from carcinoma patients contained DNA with a specific base substitution at the first or at the second position of codon 12. The base sequence of codon 13 was not affected in any of the samples.

\section{Discussion}

In the present study, a simple, reliable, and highly sensitive technique for the detection of mutant KRAS

Table 1. KRAS Codons 12 and 13 of siPCR-Processed DNA from Pancreatic Juice ${ }^{a}$

\begin{tabular}{|c|c|c|c|}
\hline \multicolumn{2}{|c|}{ Chronic pancreatitis } & \multicolumn{2}{|r|}{ Carcinoma } \\
\hline 1 & GGT GGC & 1 & 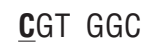 \\
\hline 2 & GGT GGC & 2 & GCT GGC \\
\hline 3 & GGT GGC & 3 & $\underline{\mathbf{C}} \overline{\mathrm{G}} \mathrm{GGC}$ \\
\hline 4 & CGT GGC & 4 & CGT GGC \\
\hline 5 & $\overline{\mathrm{G}} \mathrm{GT} \mathrm{GGC}$ & 5 & $\overline{\mathrm{G}} \mathrm{CT} \mathrm{GGC}$ \\
\hline 6 & GGT GGC & 6 & GIT GGC \\
\hline 7 & GGT GGC & & \\
\hline 8 & GGT GGC & & \\
\hline 9 & GGT GGC & & \\
\hline
\end{tabular}

SiPCR, subtractive iterative polymerase chain reaction.

${ }^{a}$ Wild-type sequence GGT GGC. alleles in the presence of an excess of wild-type alleles is described. The detection of small amounts of mutant alleles is achieved by removal of wild-type alleles by differential hybridization to wild-type specific oligonucleotides (Fig. 1A). Because hybridization takes place at the estimated melting temperature $\left(T_{m}\right)$ of the hybrid of the wild-type specific oligonucleotide and its complementary sequence, mutant alleles predominantly remain unbound, whereas wild-type alleles preferentially hybridize to the oligonucleotides. In a previous study, the removal of wild-type alleles by complementary oligonucleotides was applied to the enrichment of mutant alleles of three tumor genes harboring a variety of base substitutions (Nollau et al, 1999). In the study presented here, an iteration of the procedure is described that significantly increases the sensitivity of the method. A second step of amplification and wild-type subtraction results in a strong signal of the mutant DNA (Fig. 1B, Lane 2) that was barely detectable after the first round of subtraction (Lane 1). Corresponding results were obtained with a point-mutated P53 allele (data not shown), indicating that the method performs with equal efficiency and discrimination for the detection of mutant alleles of different sequences. Samples with a mutant-to-wildtype DNA ratio of 1:1000 can be analyzed following this two-step protocol. In theory, by iterating this approach, the enrichment of mutant alleles increases exponentially with each round of the siPCR.

The sensitivity of the siPCR assay is limited by the error rate of the DNA polymerase only. Errors of the polymerase affect both the subtraction of wild-type alleles and the detection of mutations by DNA sequencing. The percentage of fragments with base substitutions caused by polymerase errors depends on the average error rate, the fragment length, and the number of PCR cycles. In pancreatic cancer, mutations in the KRAS gene are restricted to positions 1 and 2 in codon 12. Base substitutions are verified by DNA sequencing. Assuming an error rate of the Pfu DNA polymerase of $1.3 \times 10^{-6}$ (Cline et al, 1996), twenty cycles in the first PCR would result in a fraction of $1.3 \times 10^{-5}$ alleles in which a base is substituted at a defined position (Reiss et al, 1990). The subtraction of wild-type alleles reduces the number of fragments with polymerase errors in the subsequent PCR reactions. Our previous results regarding the efficiency of absorption suggest that approximately $99 \%$ of the wild-type alleles are bound to the immobilized capture oligonucleotides. It follows that, by one absorption step, the fraction of wild-type alleles potentially modified by polymerase errors is reduced by a factor of $10^{-2}$. Thus, compared with the percentage of wildtype alleles modified in the first PCR, the percentages modified in the following PCR reactions are negligible. Alleles with erroneous base substitutions which pass the immobilized 20 mer oligonucleotides because of base substitutions outside of positions 1 and 2 of codon 12 may be modified at these positions in the PCR following the absorption. Compared with the fraction of wild-type alleles modified in the first PCR, this fraction is also negligible. In conclusion, the errors 
introduced into either base 1 or base 2 in codon 12 of the wild-type fragment in the first PCR reaction limit the sensitivity of the method. Based on the theoretical consideration above $\left(1.3 \times 10^{-5}\right.$ alleles in which a base is substituted at a defined position), the limit of sensitivity of the siPCR is calculated to 1 mutant allele in about 77,000 normal alleles. In this case the number of naturally mutated fragments would correspond to the number of fragments artificially mutated in a single nucleotide position. In practice, it should be possible to detect 1 mutant allele in 10,000 wild-type alleles unambiguously.

In addition to reducing the percentage of wild-type alleles potentially modified by polymerase errors, the subtraction of wild-type alleles is essential to make possible the detection of rare mutants in the sequencing reaction. For example, if 1 mutant allele is present in 10,000 wild-type alleles, the wild-type alleles must be reduced by a factor of about $10^{-5}$ to give an unequivocal signal in the sequencing reaction. Considering the sensitivity limit set by errors of the Pfu polymerase, three absorption steps would be sufficient to unambiguously differentiate a mutant base from the wild-type base by DNA sequencing.

In comparison with other methods aimed at the detection of mutant KRAS alleles in the presence of an excess of wild-type alleles, the siPCR protocol has some distinct advantages. Detection of mutant PCR clones by allele-specific oligonucleotide hybridization (Sidransky et al, 1992), though it pioneered the field, is laborious, technically demanding, and, for these reasons, not suitable for routine purposes. Allele-specific PCR requires multiple PCR primers complementary to the different oncogenic base substitutions (SmithRavin et al, 1995). Digital PCR requires multiple PCR and hybridization reactions (Vogelstein and Kinzler, 1999). Because single DNA molecules are amplified, DNA polymerase errors may present a problem. In contrast to these methods, few reagents are needed in the siPCR: eg, one set of primers and a device containing immobilized oligonucleotides. The siPCR results presented here confirm the finding of previous studies that in most carcinomas of the exocrine pancreas (Almoguera et al, 1988; Smit et al, 1988) and in most pancreatic juice samples from carcinoma patients (Miki et al, 1993; Tada et al, 1993; Watanabe et al, 1993), KRAS mutations in codon 12 are present. Base substitutions at the first or the second position of codon 12 were detected in each of the six carcinoma samples (Table 1). The finding of a KRAS mutation in one of nine samples from patients with chronic pancreatitis is not surprising because such mutations are also supposed to occur in nonmalignant lesions of the pancreas (Kimura et al, 1999). The siPCR results are based on the DNA sequence analysis as a definite method of confirming a specific base substitution. Along with this detection method, the high fidelity of DNA amplification performed by a polymerase with proofreading activity (Pfu polymerase) supports the specificity of a siPCR assay. In a number of corresponding investigations, the mutant-enriched PCR has been applied (Kimura et al, 1999). DNA poly- merases with proofreading activity cannot be used in this technique because the mismatch primers would be digested (Chen and Viola, 1991; Kahn et al, 1991). In consequence, polymerase errors may produce base substitutions within the recognition site of the restriction enzyme and, in this way, may cause false positive results in the RFLP analysis when many PCR cycles are performed to reach maximum sensitivity (Nollau and Wagener, 1997).

In conclusion, the siPCR assay is suitable for the sensitive detection of KRAS mutations in the pancreatic juice from patients with pancreatic carcinoma. In ongoing studies, the applicability of siPCR to the detection of mutant KRAS alleles in other samples, such as stool, is being evaluated. As reasoned recently, the method may be extended to the detection of other mutations not known a priori. To reach this goal, one would apply multiple parallel devices with immobilized complementary oligonucleotides covering the sequence range of a gene in which mutations are expected (Nollau et al, 1999). After having passed the parallel devices, the nonbound fractions would be pooled, reamplified, and submitted to a second absorption step. Our preliminary results indicate that this approach is feasible.

\section{Materials and Methods}

\section{Patients and Sample Collection}

Approximately $1 \mathrm{ml}$ of secretin-stimulated pancreatic juice was collected during routinely performed endoscopic retrograde cholangiopancreatography from patients who were clinically suspected of a pancreatic disease. Samples were stored at $-80^{\circ} \mathrm{C}$ until DNA isolation was started. Diagnosis relied on the pathological findings, the results of diagnostic routines, and/or the clinical history of the patient.

\section{Nucleic Acid Isolation}

DNA was isolated from $200 \mu$ l of pancreatic juice using commercial DNA isolation columns (QIAamp DNA Blood Kit; Qiagen, Hilden, Germany). To establish the repetitive enrichment procedure, mutant DNA containing a $\mathrm{G} \rightarrow \mathrm{T}$ exchange at the first position in codon 12 of the KRAS gene was isolated from CaLu-I cells and mixed with wild-type DNA from MCF7 cells in defined mutant-to-wild-type ratios as described (Nollau et al, 1999).

\section{Polymerase Chain Reactions and ssDNA Preparation}

In the first PCR, 100 ng of the mutant KRAS DNA mixture or $5 \mu \mathrm{l}$ of the DNA preparation from pancreatic juice was amplified in a $50-\mu$ l reaction with $0.3 \mu \mathrm{mol} / / \mathrm{l}$ of primers forward (5'-AACCTTATGTGTGACATGTTC-3') and reverse-1 (5'-biotin-ATGGTCAGAGAAACCTTTAT-3'), and $2.5 \cup$ of $P f u$ DNA polymerase under the conditions recommended by the supplier (Stratagene, Heidelberg, Germany). The second PCR was performed with $5 \mu$ l of the fourth flow-through fraction using the primers forward and reverse-2 (5'-biotin-TCAAAGAATGGTCCTGCACC-3'). 
Polymerase chain reactions were performed on a thermal cycler (Biozym, Oldenhausen, Germany) with an initial denaturation at $94^{\circ} \mathrm{C}$ for 5 minutes, 20 cycles of 1 minute at $51^{\circ} \mathrm{C}$ (first PCR) or $59^{\circ} \mathrm{C}$ (second PCR), 1 minute at $72^{\circ} \mathrm{C}$, and 1 minute at $94^{\circ} \mathrm{C}$, followed by a final extension at $72^{\circ} \mathrm{C}$ for 10 minutes. ssDNA was prepared and quantified as described (Nollau et al, 1999).

\section{Column Preparation and Chromatographic Procedure}

A column containing the oligonucleotide capture (5'biotin-GCCTACGCCACCAGCTCCAA-3') complementary to codons 9 to 15 of the KRAS wild-type allele was prepared and perfused with hybridization buffer as described (Nollau et al, 1999). After equilibration of the column at the calculated (Wetmur, 1991) $\mathrm{T}_{\mathrm{m}}$ of $37^{\circ} \mathrm{C}, 1$ $\mu \mathrm{l}$ of ssDNA preparation diluted in $100 \mu \mathrm{l}$ of hybridization buffer was applied to the column. With a constant flow rate of $0.1 \mathrm{ml}$ per minute, the first milliliter of buffer was discarded and the following flow-through was collected in 500- $\mu$ l portions. The nonbound fractions were desalted by spin cartridges (QIAquick PCR Purification, Qiagen) as recommended by the manufacturer.

\section{RFLP Analysis and DNA Sequencing}

For RFLP analysis, a PCR was performed as described with $10 \mu \mathrm{l}$ of the nonbound fraction and the primers RFLP forward (5'-ACTGAATATAAACTTGTGGTAGTTGGACCT$\left.3^{\prime}\right)$ and reverse-2. The primer RFLP forward introduces an artificial BstNI restriction site at codon 12 allowing the detection of mutations by RFLP analysis as described (Nollau et al, 1999). PCR products were digested with the restriction enzyme BstNI as recommended by the supplier (NEB, Schwalbach/Taunus, Germany) and electrophoresed in $40 \mathrm{~g} / \mathrm{l}$ of lowmelting-point agarose gels. DNA sequencing was performed with the primer reverse-2 by an ABI 373A DNA Sequencer (Applied Biosystems, Weiterstadt, Germany) using the manufacturer's Taq cycle sequencing protocol.

\section{References}

Almoguera C, Shibata D, Forrester K, Martin J, Arnheim N, and Perucho $M$ (1988). Most human carcinomas of the exocrine pancreas contain mutant $\mathrm{c}-\mathrm{K}-$ ras genes. Cell 53 : 549-554.

Chen J and Viola MV (1991). A method to detect ras point mutations in small subpopulations of cells. Anal Biochem 195:51-56.

Cline J, Braman JC, and Hogrefe HH (1996). PCR fidelity of pfu DNA polymerase and other thermostable DNA polymerases. Nucleic Acids Res 24:3546-3551.

Fuery CJ, Impey HL, Roberts NJ, Applegate TL, Ward RL, Hawkins NJ, Sheehan CA, O‘Grady R, and Todd AV (2000). Detection of rare mutant alleles by restriction endonucleasemediated selective-PCR: Assay design and optimization. Clin Chem 46:620-624.

Kahn SM, Jiang W, Culbertson TA, Weinstein IB, Williams GM, Tomita N, and Ronai Z (1991). Rapid and sensitive nonradioactive detection of mutant K-ras genes via "enriched" PCR amplification. Oncogene 6:1079-1083.
Kimura W, Zhao B, Futakawa N, Muto T, and Makuuchi M (1999). Significance of K-ras codon 12 point mutation in pancreatic juice in the diagnosis of carcinoma of the pancreas. Hepatogastroenterology 46:532-539.

Miki H, Matsumoto S, Harada H, Mori S, Haba R, Ochi K, Kobayashi S, and Ohmori M (1993). Detection of c-Ki-ras point mutation from pancreatic juice. A useful diagnostic approach for pancreatic carcinoma. Int J Pancreatol 14:145148.

Nollau P, Fischer C, Tschentscher P, and Wagener C (1999). Enrichment of mutant alleles by chromatographic removal of wild type alleles: A new principle for the detection of alleles with unknown point mutations at excess of wild type alleles. Clin Chem Lab Med 37:877-881.

Nollau P and Wagener C (1997). Methods for detection of point mutations: Performance and quality assessment. IFCC Scientific Division, Committee on Molecular Biology Techniques. Clin Chem 43:1114-1128.

Reiss J, Krawczak M, Schloesser M, Wagner M, and Cooper DN (1990). The effect of replication errors on the mismatch analysis of PCR-amplified DNA. Nucleic Acids Res 18:973978.

Sidransky D (1997). Nucleic acid-based methods for the detection of cancer. Science 278:1054-1059.

Sidransky D, Tokino T, Hamilton SR, Kinzler KW, Levin B, Frost P, and Vogelstein B (1992). Identification of ras oncogene mutations in the stool of patients with curable colorectal tumors. Science 256:102-105.

Smit VT, Boot AJ, Smits AM, Fleuren GJ, Cornelisse CJ, and Bos JL (1988). KRAS codon 12 mutations occur very frequently in pancreatic adenocarcinomas. Nucleic Acids Res 16:7773-7782.

Smith-Ravin J, England J, Talbot IC, and Bodmer W (1995). Detection of c-Ki-ras mutations in fecal samples from sporadic colorectal cancer patients. Gut 36:81-86.

Somers VA, Leimbach DA, Murtagh JJ, and Thunnissen FB (1998). Exonuclease enhances hybridization efficiency: Improved direct cycle sequencing and point mutation detection. Biochim Biophys Acta 1379:42-52.

Somers VA, Moerkerk PT, Murtagh JJ Jr, and Thunnissen FB (1994). A rapid, reliable method for detection of known point mutations: Point-EXACCT. Nucleic Acids Res 22:48404841.

Tada M, Omata M, Kawai S, Saisho H, Ohto M, Saiki RK, and Sninsky JJ (1993). Detection of ras gene mutations in pancreatic juice and peripheral blood of patients with pancreatic adenocarcinoma. Cancer Res 53:2472-2474.

Vogelstein B and Kinzler KW (1999). Digital PCR. Proc Natl Acad Sci USA 96:9236-9241.

Watanabe H, Sawabu N, Ohta H, Satomura Y, Yamakawa O, Motoo Y, Okai T, Takahashi H, and Wakabayashi T (1993). Identification of K-ras oncogene mutations in the pure pancreatic juice of patients with ductal pancreatic cancers. Jpn J Cancer Res 84:961-965.

Wetmur JG (1991). DNA probes: Applications of the principles of nucleic acid hybridization. Crit Rev Biochem Mol Biol 26:227-259. 